\title{
Metodología para Estimación de Energía Firme a través de Series Hídricas Sintéticas Desacopladas
}

\author{
Yurany A. Osorno-Cardona ${ }^{1}$, Diego A. Mejia-Giraldo², Nicolás Muñoz-Galeano² \\ (1) XM S.A. E.S.P. - Filial de ISA, Calle 12 Sur No. 18-168, Medellín - Colombia \\ (e-mail:yosorno@xm.com.co) \\ (2) Grupo de Investigación GIMEL, Departamento de Ingeniería Eléctrica, Facultad de Ingeniería, \\ Universidad de Antioquia, Calle 67 No.53-108, Medellín - Colombia (e-mail: diego.mejia@udea.edu.co; \\ nicolas.munoz@udea.edu.co)
}

Recibido Ene. 4, 2018; Aceptado Mar. 21, 2018; Versión final Jun. 1, 2018, Publicado Oct. 2018

\begin{abstract}
Resumen
Este artículo propone una variante a la metodología oficial colombiana para el cálculo de la energía firme (EF) de plantas de generación hidráulica (MCREG). La EF es la máxima energía eléctrica que es capaz de suministrar una planta de generación continuamente en condiciones de baja hidrología. En este trabajo, se plantea que, para determinar la EF, las series hídricas se modelen desacopladas anualmente para no suponer la ocurrencia de eventos hidrológicos futuros en el mismo orden cronológico histórico. Las series son generadas a partir de datos históricos de caudales empleando distribuciones normales correlacionadas. Aparte de la creación de series que reflejan la tendencia histórica, se ha logrado modelar la correlación temporal entre los caudales hídricos mensuales de plantas hidráulicas. La metodología desacoplada propuesta (abreviada como MDEF) se compara con la MCREG para dos plantas hidráulicas (338 y 1000 MW), y los resultados indican que la EF obtenida con la MDEF es mayor y menos incierta si el número de series sintéticas es significativo.
\end{abstract}

Palabras clave: caudal hídrico; distribuciones normales correlacionadas; energía firme; generación hidráulica; series hídricas históricas

\section{Methodology for the stimation of Firm Energy through Desacopled Hidrical Synthetic Series}

\begin{abstract}
This paper proposes a variant of the Colombian official methodology employed in the computation of firm energy (FE) produced by hydro power plants (MCREG). Firm energy is defined as the maximum electrical energy that a plant is able to continuously deliver under low hydrological conditions. This research establishes that for determining the FE, hydrological series should be annually decoupled in the MCREG optimization model to avoid the assumption that future hydrological events will occur in the same historical chronological order. Time series are randomly generated through multivariate and correlated normal distributions, whose parameters are estimated from historical inflow data. Additionally, it is also possible to model the intertemporal correlation between monthly inflows of hydro power plants. The proposed methodology (abbreviated as MDEF) is compared with MCREG. This comparison was done for two power plants (338 and $1000 \mathrm{MW}$ ) and the results indicate that EF obtained with MDEF is higher and less uncertain if a significant number of time series is considered.
\end{abstract}

Keywords: water inflows; multivariate normal distribution; firm energy; hydro generation; inflow time series 
Metodología para Estimación de Energía Firme a través de Series Hídricas Sintéticas $\quad$ Osorno-Cardona

\section{INTRODUCCIÓN}

Los mercados de energía de los diferentes países se han centrado en dar confiabilidad a los sistemas de potencia, a través de mecanismos de capacidad que incentivan la inversión en proyectos de expansión en el sector eléctrico (Laurens y Petra, 2008; Choong-Kyo et al., 2011). Dichos mecanismos garantizan que en condiciones de escasez se tenga suficientes recursos energéticos para abastecer la demanda a un precio razonable (Lennar, 2010). Los mecanismos de capacidad permiten gestionar el riesgo financiero a través de obras de generación que estén acordes con el crecimiento variable de la demanda. Las características de cada mercado determinan cual es el mejor mecanismo de capacidad que se debe elegir según el tipo de parque de generación y la capacidad de inversión. En (Hasani et al., 2011; Huber et al., 2006); se presentan algunos tipos de mecanismos que son utilizados en diferentes países, por mencionar algunos: el cargo por confiabilidad o pago por capacidad es empleado en Argentina, Chile, Perú y Colombia; el mercado de capacidad y los requerimientos de capacidad son mecanismos utilizados en estados Unidos; mientras que los contratos de capacidad son utilizados en Brasil. Todos los mecanismos de capacidad utilizan como principal insumo el concepto de Energía Firme (EF), por lo que es tema de investigación en este artículo.

En (Hreinsson y Barroso, 2004) se define la EF como la cantidad máxima de energía que el sistema hidroeléctrico puede producir bajo la condición hidrológica más adversa de los registros históricos. El concepto de EF es utilizado por los países como mecanismo de confiabilidad en sus sistemas de potencia, según las condiciones de sus mercados de energía. El continente americano se caracteriza por tener recursos hídricos, los cuales se ven afectados por condiciones críticas de abastecimiento de agua. En Brasil, por ejemplo, predominan los recursos hídricos (Bezerra et al., 2010). Brasil cuenta con un mecanismo financiero para cubrir los riesgos hidrológicos que afectan a los generadores hidráulicos (De Yagi, 2012). La EF de una planta hidroeléctrica es el principal insumo de este mecanismo y equivale a la producción media de la planta a lo largo de un periodo crítico. Un periodo crítico se considera como aquel en el que se presenta la disminución excesiva del volumen de los embalses, resultado de una temporada de desabastecimiento de agua.

En Perú, según (Res. Oslnergmln 036, 2015), la EF se calcula para las plantas de generación hidroeléctrica como la máxima producción esperada de energía eléctrica determinada para una probabilidad de excedencia (PE) de 95\%. En (Yagi, 2012) se menciona el cálculo de la EF de Argentina, Chile y Estados Unidos. En Argentina, la EF se determina usando un nivel del $70 \%$ PE para la planta y es el valor máximo de energía para los contratos anuales que pertenecen a las plantas hidroeléctricas. En Chile, la EF se define como la energía anual para una condición hidrológica critica con el 90\% PE en el sistema. En los Estados Unidos, la "Bonneville Power Administration", la EF es considerada como la energía eléctrica disponible producida por el sistema hidroeléctrico en condiciones críticas y está disponible en cantidades que varían dependiendo de las condiciones de la temporada y el clima. Particularmente, para los países del continente americano, los sistemas eléctricos pueden ser afectados por las condiciones críticas de abastecimiento de agua (periodo de escasez). Esto ocasiona altos incrementos en los costos de generación en países donde predomina la generación hidráulica (Aurelie et al., 2013). En un periodo de escasez, la generación mediante energía hidráulica debe ser disminuida para garantizar los niveles mínimos de los embalses. Esto obliga a que se genere energía a través de otras tecnologías como por ejemplo térmica o nuclear, las cuales encarecen la operación del sistema dado que sus costos de operación son más altos y/o las tecnologías son menos eficientes.

Además del interés del uso de EF en los mercados de energía, la metodología para el cálculo de EF también ha sido objeto de investigación. Por ejemplo, en (Jaramillo, et. al., 2008) se proponen dos metodologías alternativas para mejorar el cálculo de la EF de las plantas de generación hidráulicas, con el fin de obtener una mayor asignación de EF y un beneficio económico que se traslada al usuario final. En (Faria, et al., 2009) se explican diferentes métodos de asignación de la EF para sistemas en cadena, que buscan obtener el valor de EF óptimo a pesar de que el concepto de EF es usado en el contexto internacional, este artículo se enfoca en el ámbito colombiano; sin embargo, la metodología de este artículo pueden ser usados en cualquier país que utilice algún mecanismo de capacidad para garantizar la confiabilidad del sistema. En Colombia desde 2006 se aplica el concepto de EF para plantas hidráulicas. De hecho estas representan el 69.93\% de la capacidad instalada del país (UPME, 2016). La EF es remunerada a los agentes generadores a través del mecanismo de capacidad Cargo por Confiabilidad CxC, (Res. CREG 071, 2006). En este mecanismo el agente generador debe garantizar cumplimiento de la obligación de EF adquirida a través de un mecanismo de subasta o de algún tipo de asignación de energía. La metodología oficial regulada en Colombia por la Comisión de Regulación y Energía Gas - CREG, conocida como metodología CREG (MCREG), está estructurada por un modelo de optimización que maximiza la mínima energía producida por la planta de generación para todos los años con registros históricos disponibles de caudales hídricos mensuales. 
El interés del cálculo de la EF no solo se aplica al caso de la tecnología de generación hidráulica, también puede aplicarse a cualquier tecnología de generación. Por mencionar algunas y particularizando a las nuevas tecnologías emergentes basadas en recurso renovable: 1) Generación eólica, el cálculo de la EF en Colombia puede realizarse a partir de la resolución CREG 167 de 2017 (Res. CREG 167 de 2017), en la resolución se detalla la metodología cuando se dispone o no de por lo menos diez años de medida para la velocidad del viento. 2) Generación solar fotovoltaica, el cálculo de la EF en Colombia puede realizarse a partir de la resolución CREG 201 de 2017 (Res. CREG 201 de 2017), la resolución indica que para poder declarar EF se debe disponer de información de radiación horizontal y temperatura ambiente de por lo menos diez años. Para los casos de energía eólica y solar, la EF no es calculada a través de modelos de optimización ni acople anual como en el caso de la EF hidráulica; por lo tanto, la propuesta presentada en este trabajo sobre desacople temporal no aplica, pues es diseñada para modelos de optimización de EF como el caso hidráulico. Por ejemplo, la metodología que se plantea en este trabajo podría ser extendida al caso de modelos empleados en investigación en Brasil como los presentados en (Yagi, 2012), (Faria, et al., 2009). En estos artículos la EF es basada en conceptos similares al colombiano en el que se construye un modelo de maximización de EF empleando los datos históricos disponibles de hidrologías de manera acoplada.

En este artículo se propone un desacople temporal en el manejo de las series hídricas que consiste en considerar de manera independiente el orden de ocurrencia de cada una de las series hídricas históricas. A esta metodología se le denominará metodología desacoplada de energía firme (MDEF). Además, la MDEF plantea la construcción de escenarios hídricos a partir de registros históricos de caudales usando distribuciones normales correlacionadas para realizar una estimación de EF con menor incertidumbre. La MDEF se aplica en el caso de dos plantas de generación hidráulicas en Colombia de 338 MW y de 1000 MW.

\section{METODOLOGÍA PROPUESTA PARA EL CÁLCULO DE LA EF (MDEF)}

La EF para plantas hidráulicas en Colombia es calculada según criterios del regulador CREG a través de un modelo de programación lineal entera mixta como se explica en el anexo 9 de Res. CREG 071 de 2006 . La MCREG modela las características propias de la planta hidráulica bajo estudio para cada mes y para cada uno de los años a evaluar. El número de años a evaluar corresponde a los años para los cuales existen registros históricos de caudales. También, para estimar la EF se modelan las restricciones operativas hídricas habituales como balances de agua y límites de almacenamiento de flujos de salida. La función objetivo es la maximización de la EF de todos los años considerados en el análisis.

La MCREG entrega un valor de EF para cada una de los años en el horizonte de análisis. Posteriormente, una distribución de probabilidad para la EF anual debe ser calculada usando el procedimiento indicado en (Manual HIDENFICC, 2007). La distribución se construye ordenando los valores de EF de menor a mayor, el menor valor corresponde al $100 \%$ probabilidad de excedencia -PE-y el mayor valor corresponde al $0 \%$ PE. Las magnitudes de EF que se utilizan para las subastas del cargo por confiabilidad en Colombia son la EF al $100 \%$ PE conocida como EF base y la EF al 95\% PE. Este último valor corresponde al percentil 5 de la distribución construida.

La propuesta de este trabajo consiste en utilizar series hídricas de manera desacoplada en la MCREG y construir múltiples series hídricas sintéticas a partir de registros históricos de caudales mensuales. Actualmente, la MCREG considera el orden cronológico de cada una de las series de caudales hídricos históricos, es decir, replica la historia para estimar la EF de una planta de generación hidráulica. La MCREG únicamente emplea series hídricas históricas para estimar la distribución de probabilidad de EF de una planta, lo cual es una desventaja dado el bajo número de escenarios a considerar. Para ganar independencia en el orden de ocurrencia de las series, en este trabajo se plantea desacoplar el modelo matemático de la CREG. Esta filosofía, que además de permitir evaluar múltiples condiciones hidrológicas, elimina el modelamiento de la ocurrencia de eventos hidrológicos en el mismo orden cronológico histórico. Por otro lado, con el fin de realizar una mejor estimación de la EF, también se plantea un procedimiento para construir series hídricas sintéticas a partir de registros históricos.

En la Fig. 1a), se muestra la representación del manejo de las series de caudales hídricos ordenadas cronológicamente según la historia. Las líneas verticales punteadas muestran el inicio de cada uno de los cuatro años ilustrados. En esta se observa la forma en que las series son tratadas tanto en MCREG como en la estrategia planteada. En este trabajo, el horizonte de análisis del modelo de optimización para estimar la EF es de un año como se muestra en la Fig. 1b). Esta estrategia permite calcular una EF para cada año independiente de las series hídricas disponibles. De hecho, es posible también adicionar series sintéticas que el regulador o el planeador desee evaluar para determinar el impacto en la EF resultante. 


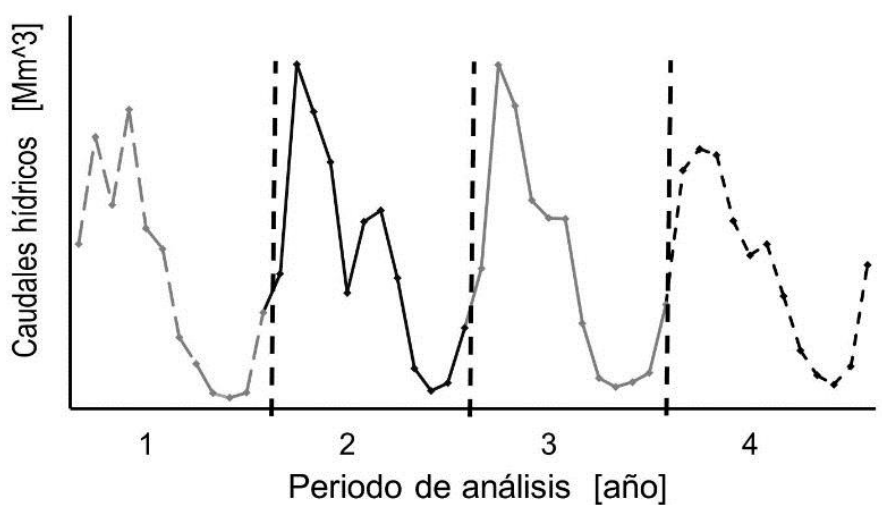

a) Manejo series hídricas - MCREG.

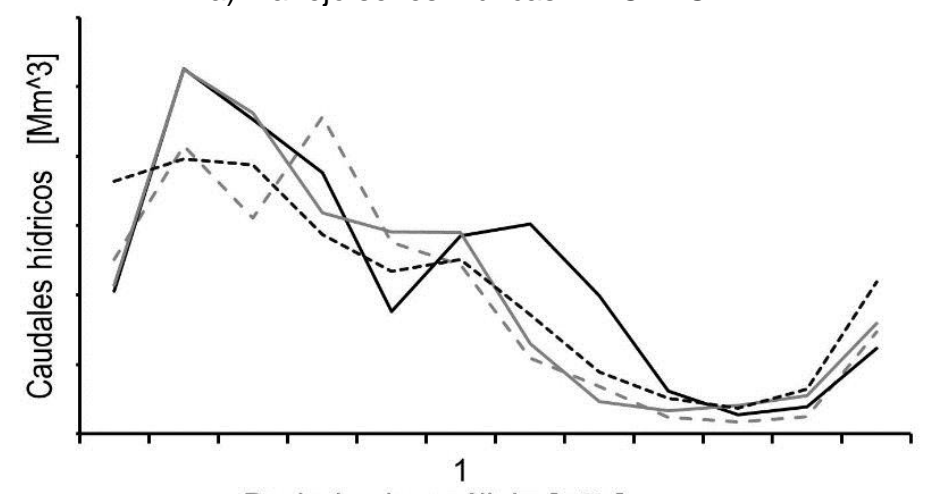

Periodo de análisis [año]

b) Manejo series hídricas - MDEF.

Fig. 1. Series hídricas.

\section{Modelo matemático propuesto}

El modelo modificado, inspirado en la MCREG (anexo 9, Res. CREG 071 de 2006), para el cálculo de la EF se presenta en las ecuaciones (1)-(22). La ecuación (1) representa la función objetivo, esta consiste en maximizar la EF de la serie $w$, denominada $E F_{w}$. La ecuación (2) garantiza que $E F_{w}$ sea la cota inferior de la producción mensual de energía ante la serie hídrica dada por $q_{m, w}$. $\rho$ es el coeficiente medio de producción de los recursos de generación en $(\mathrm{MWh} / \mathrm{m} 3)$. El coeficiente de producción de una planta refleja cómo cada unidad de entrada a través de la turbina se convierte en energía eléctrica. El parámetro $\sigma_{m}$ es el número de días en el mes $m$. La ecuación (3) representa el balance hídrico en la que se actualiza el volumen del embalse $\varepsilon_{m}$ para el mes $m$ teniendo en cuenta los caudales $q_{m, w}$ de la serie $w$, la descarga de agua (caudales turbinados) $\tau_{m}$ y el vertimiento $v_{m}$. Las restricciones en (4) representan los límites de volumen mínimo $\varepsilon_{\min }$ y de volumen máximo $\varepsilon_{\text {máx. }}$. Las restricciones (5)-(8) se aplican cuando la planta tiene curva guía mínima de volumen $\varepsilon_{(\text {CGmín)m }}$ y curva guía máxima de volumen $\varepsilon_{(\text {CGmax }) m}$ para sus embalses (MME y Urrá S.A. E.S.P., 2011). El objetivo de la curva guía es minimizar el riesgo ante la amenaza natural de desbordamientos de ríos aguas abajo del embalse. De hecho, la curva guía máxima controla las crecientes en el embalse y la curva guía mínima tiene en cuenta las restricciones ambientales. Las variables binarias $w_{m}, y_{m}$ y $z_{m}$ controlan que el volumen esté dentro de los límites permitidos para cada planta y se activan en las ecuaciones (9) y (13). La ecuación (13) aplica cuando la planta tiene restricciones de curva guía mínima $\varepsilon_{\text {(CGmín)m }}$, condición identificada cuando la variable de control $z_{m}=1$, de lo contrario si $z_{m}=0$ es porque a la planta no le aplica esta restricción. Las ecuaciones (10) y (11) representan los límites para el vertimiento $v_{m} . u_{m}$ es una variable de decisión binaria que indica el mes en el que se presenta vertimiento. Si $u_{m}=0$, el $v_{m}$ es forzado a ser cero y $\tau_{m}$ está condicionado por los límites de la turbina (entre 0 y el $\tau_{\text {max }_{m}}$ ) como sugiere la ecuación (12). Si $u_{m}=1$, se permite que el $v_{m}$ se encuentre entre sus límites de vertimiento mínimo $v_{\min _{m}}$ y de vertimiento máximo $v_{\max }$, de acuerdo con las ecuaciones (10) y (11); el turbinamiento $\tau_{m}$ debe ser al menos un valor tal que garantice disminuir el volumen desde su valor máximo hasta su valor mínimo en un mes como se indica en las ecuaciones (14)-(16). Las ecuaciones (17)-(19) se emplean para relajar la descarga de agua mínima de la turbina. Si $x_{m}=1$, la descarga de agua puede ser una cantidad $\tau_{m}$ menor a $\tau_{m i ́ n}$; de lo contrario, la descarga de agua debe estar dentro de los límites normales $\tau_{m i ́ n}$ y $\tau_{\max _{m}}$. Esta restricción se utiliza para considerar la condición crítica de abastecimiento de agua más adversa que puede presentarse en un periodo de escasez, algunas plantas hidroeléctricas no son capaces de mantener un turbinamiento mínimo $\tau_{m i ́ n}$ por las condiciones de la temporada, por lo que es necesario 
que la descarga sea inferior $\tau_{m i n}$. Cuando la variable binaria $i_{m}=1$, el volumen ha alcanzado su valor máximo (ecuación (20)), que también obliga a $u_{m}$ a ser 1 según la ecuación (21). Si $i_{m}=0$, el volumen del embalse puede estar dentro de sus límites normales. La ecuación (22) indica que el vertimiento máximo es el valor que puede reducir el volumen del embalse a cero en un mes.

Maximizar $E F_{w}$

Sujeto a:

$E F_{w}-\bar{\rho} * \tau_{m} * \frac{1}{\sigma_{m}} 1000 \leq 0$

$\varepsilon_{m}=\varepsilon_{m-1}+q_{m, w}-\tau_{m}-v_{m}$

$\varepsilon_{\min } \leq \varepsilon_{m} \leq \varepsilon_{m a ́ x}$

$\varepsilon_{\min } \leq \varepsilon_{m}-\left[\varepsilon_{(\text {CGmax }) m}-\varepsilon_{\min }\right] . w_{m}$

$\varepsilon_{m}-\left[\varepsilon_{\max }-\varepsilon_{(\text {CGmax }) m}\right] \cdot y_{m} \leq \varepsilon_{\min }$

$\varepsilon_{(\text {CGmin }) m} \leq \varepsilon_{m}+\left[\varepsilon_{(\text {CGmin }) m}-\varepsilon_{\text {min }}\right] . z_{m}$

$\varepsilon_{m}+\left[\varepsilon_{\text {max }}-\varepsilon_{(\text {CGmin }) m}\right] \cdot z_{m} \leq \varepsilon_{\max }$

$y_{m}-w_{m} \leq 0$

$v_{m}-v_{\min _{m}} \cdot u_{m} \geq 0$

$v_{m}-v_{\max _{m}} \cdot u_{m} \leq 0$

$0 \leq \tau_{m} \leq \tau_{\max m}$

$\tau_{m}+\tau_{\max _{m} \cdot z_{m}} \leq \tau_{\max _{m}}$

$\tau_{m} \geq u_{m} . \operatorname{mín}\left\{\left(\left(\varepsilon_{\text {máx }}-\varepsilon_{\text {mín }}\right)+q_{m, w}\right), \tau_{\text {máx }}\right\}$

$\tau_{m} \geq u_{m} \cdot \min \left\{\left(\left(\varepsilon_{\text {máx }}-\varepsilon_{(C G m i ́ n) m}\right)+q_{m, w}\right), \tau_{\max _{m}}\right\}$

$\tau_{m} \geq y_{m} \cdot \min \left\{\left(\left(\varepsilon_{(\text {CGmax }) m}-\varepsilon_{\text {min }}\right)+q_{m, w}\right), \tau_{\max _{m}}\right\}$

$\tau_{m}+\tau_{m}^{\prime} \geq \tau_{m i n}$

$\tau_{m}+\left[\tau_{\max _{m}}-\tau_{\text {mín }_{m}}\right] \cdot x_{m} \leq \tau_{m \text { áx }}$

$\tau_{m}^{\prime}-\tau_{m^{\prime} n_{m}} \cdot x_{m} \leq 0$

$\varepsilon_{m}-\left[\varepsilon_{\text {max }}-\varepsilon_{\text {min }}\right] . i_{m} \geq \varepsilon_{\text {min }}$

$u_{m}-i_{m} \geq 0$

$v_{m}+\tau_{m} \leq \varepsilon_{m-1}+q_{m, w}$

Donde $i_{m}, u_{m}, x_{m}, z_{m} \in\{1,0\}$

\section{Proceso para generar series sintéticas}

La simulación con series sintéticas de caudales hídricos consiste en generar $N$ series sintéticas a partir de los caudales históricos registrados por la planta hidráulica. Cada caudal histórico $q_{m, t}^{H}$ representa el caudal medio observado en el mes $m$ para el año $t$. Por lo tanto, si $T$ representa la cantidad de años con registros históricos de caudales, entonces $t=1, \cdots, T$. Las series sintéticas anuales de caudales son construidas empleando una distribución normal multivariada y correlacionada con media $\mu$ y matriz de covarianza $\Sigma$, es decir:

$\tilde{q} \sim N\left(\mu_{m}, \Sigma\right)$ 
En este caso, cada componente del vector $\mu=\left(\mu_{1}, \ldots \mu_{m}, \ldots, \mu_{12}\right)$, es calculada como:

$\mu_{m}=\frac{1}{T} \sum_{t=1}^{T} q_{m, t}^{H}$

Y la matriz de covarianza $\Sigma$ es estimada como:

$$
\sum=\left[\begin{array}{cccccc}
\beta_{1,1}^{2} & \beta_{1,2} & \cdots & \cdots & \cdots & \beta_{1,12} \\
\beta_{2,1} & \beta_{2,2}^{2} & \cdots & \cdots & \cdots & \beta_{2,12} \\
\vdots & \cdots & \ddots & \beta_{n, m} & \cdots & \beta_{n, 12} \\
\vdots & \cdots & \beta_{n, m} & \beta_{m, m}^{2} & \cdots & \vdots \\
\vdots & \cdots & \cdots & \cdots & \ddots & \vdots \\
\beta_{12,1} & \beta_{12,2} & \cdots & \cdots & \cdots & \beta_{12,12}^{2}
\end{array}\right]
$$

Donde la covarianza entre el caudal del mes $m$ y el mes $n$ es estimada usando:

$\beta_{m n}=\frac{1}{T-1} \sum_{t=1}^{T}\left(q_{m, t}^{H}-\mu_{m}\right)\left(q_{n, t}^{H}-\mu_{n}\right), \quad m=1, \ldots, 12 ; y n=1, \ldots, 12$

y para el caso $m=n$ se tiene que $\beta_{m m}$ es la varianza del caudal del $m$-ésimo mes.

Esta estrategia permite que las múltiples series sintéticas generadas garanticen que la tendencia mensual de la media, varianza y correlaciones satisfacen las estadísticas históricas. Una vez se han estimado los parámetros descritos anteriormente, se generan $N$ series sintéticas fruto de una realización en particular de un vector de caudales mensuales. Por lo tanto, $q_{m, w}$ almacena el caudal sintético del mes $m$ de la serie $w$.

\section{Simulación de EF}

Para cada serie $w$ el modelo ilustrado es ejecutado para obtener $E F_{w}, w=1, \ldots, N$. El procedimiento llevado a cabo es ilustrado en la Fig. 2. En datos de entrada se ingresa toda la información técnica de las plantas hidráulicas como: mínimos técnicos de volumen, vertimiento y descarga de agua; después se inicializa el contador de las series en $1, w=1$; posteriormente se ejecuta el generador de $N$ series sintéticas, el cual considera el procedimiento indicado en las ecuaciones (23)-(26). Se ejecuta el modelo ilustrado en (1)-(22) que retorna $E F_{w}$. Este proceso se repite hasta que $w=N$.

Finalmente, los resultados de EF son ordenados de menor a mayor para obtener la distribución de probabilidad, con la cual se obtiene la EF base y la EF al $95 \%$ PE.

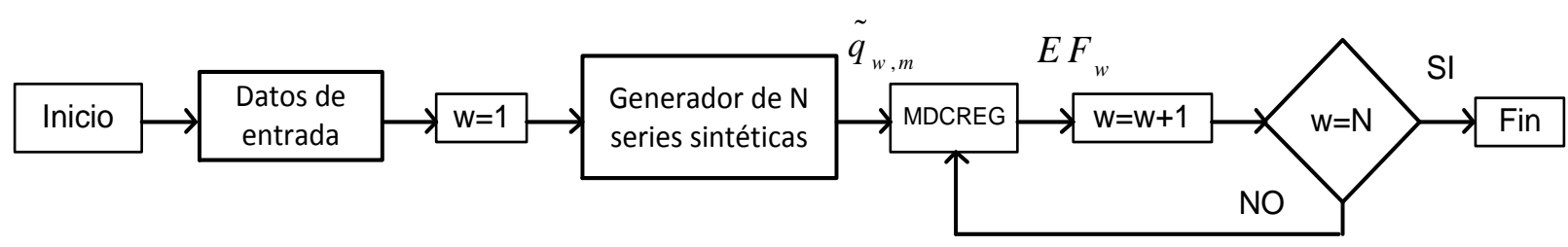

Fig. 2. Simulación de EF con series sintéticas hídricas

\section{RESULTADOS}

En esta sección se evalúa la propuesta y se realiza el análisis planteado sobre dos plantas de generación hidráulica. La Fig. 3 muestra la topología de las dos plantas de generación que fueron modeladas, la Fig. 3. a) muestra a la Planta 1 (338 MW), la cual solo es alimentada por un único río. La Fig. 3.b) muestra a la planta 2 (1000 MW), en la cual sus embalses son alimentados por 4 ríos para los cuales se cuenta con registros históricos de caudales $q_{1}-q_{4}$.

En la Tabla 1 se muestran las características técnicas de cada planta de generación hidráulica (declaración de parámetros CREG, 2011). Solo la planta 1 tiene condiciones especiales de curva guía mínima y curva guía máxima como se expone en las ecuaciones (5)-(8), (15)-(16). 


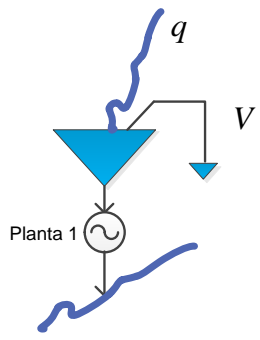

a) Planta 1



b) Planta 2

Fig. 3. Topología de las plantas de prueba

Tabla 1: Características de las plantas de generación hidráulica

\begin{tabular}{|l|l|l|l|l|l|}
\hline Características & Planta 1 & Planta 2 & Características & Planta 1 & Planta 2 \\
\hline$\varepsilon_{\min }\left[\mathrm{Mm}^{3}\right]$ & 358.97 & 20.68 & $\tau_{\max _{a, m}}\left[\mathrm{~m}^{3} / \mathrm{s}\right]$ & 383.0 & 129.4 \\
\hline$\varepsilon_{\max }\left[\mathrm{Mm}^{3}\right]$ & $1,822.63$ & 569.64 & $\tau_{\min _{a, m}}\left[\mathrm{Mm}^{3} / \mathrm{s}\right]$ & 0 & 0 \\
\hline$\varepsilon_{u t i}\left[\mathrm{Mm}^{3}\right]$ & $1,233.62$ & 548.96 & $\rho\left[\mathrm{MW} / \mathrm{Mm}^{3} / \mathrm{s}\right]$ & 0.4729 & 6.9925 \\
\hline
\end{tabular}

\section{Caudales históricos}

Las Fig. 4 a) y b) muestran el comportamiento histórico e incertidumbre de los caudales hídricos $q_{m}^{H}$ para cada mes; estas fueron construidas con caudales correspondientes a 50 años para la planta 1 y de 32 años para la planta 2. Típicamente en Colombia desde mayo hasta noviembre (estación invierno) se tienen altos caudales; y desde diciembre hasta abril (estación verano) los caudales son menores. Esta situación es observada en ambas plantas.

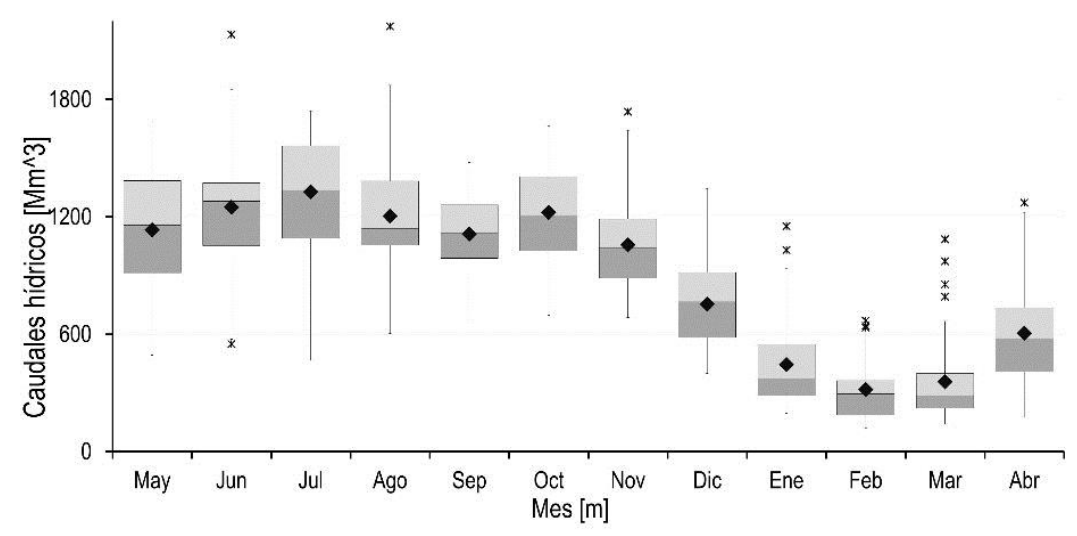

a) Planta 1

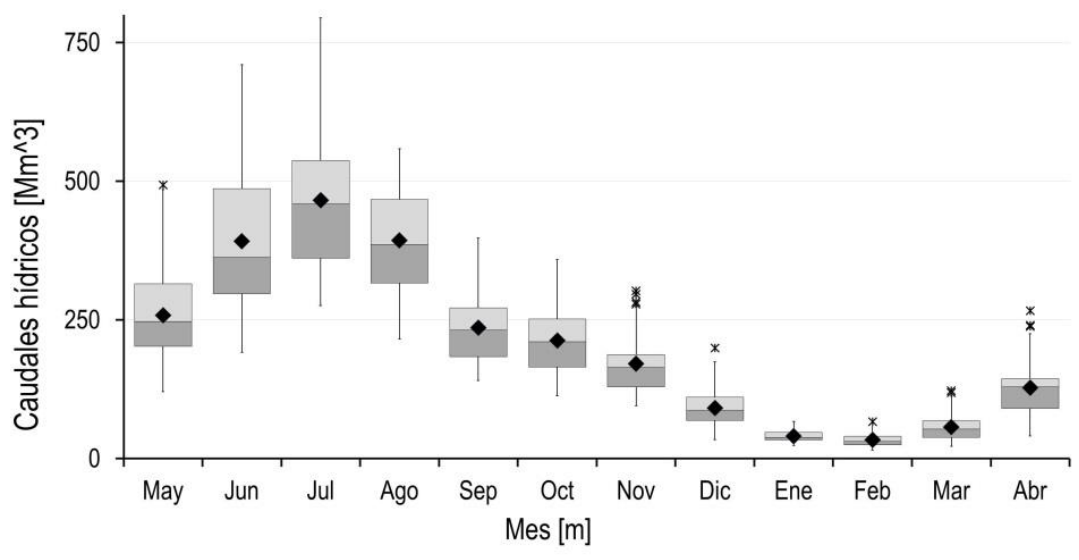

b) Planta 2

Fig. 4. Distribución de caudales hídricos históricos mensuales 
La incertidumbre mensual, según los datos, tiende a ser mayor en julio y menor en febrero. También puede observarse que hay datos atípicos por encima de la media durante los meses de noviembre, enero, febrero, marzo y abril, y por debajo para junio para la planta 1; para la planta 2 se presentan datos atípicos solo por encima de la media para los meses de noviembre, diciembre, febrero, marzo y abril. Julio se caracteriza, porque presenta el mayor caudal hídrico. En cambio, el caudal hídrico fue significativamente menor en febrero.

\section{Construcción de caudales sintéticos}

Al generar $N=1000$ series sintéticas para cada una de las plantas bajo estudio, se construyeron las distribuciones resultantes, y se presentan en las Fig. 5. a) y c). Según éstas, los caudales generados sintéticamente exhiben distribuciones mensuales similares a las distribuciones históricas. También, al tomar la media y desviación de las muestras mensuales se obtienen tendencias altamente similares a las exhibidas por los caudales históricos, según se observa en las Fig. 5 b) y d). Las diferencias presentadas fueron inferiores al $6 \%$ en desviación estándar e inferiores al $1.4 \%$ en promedio de caudales. Estos resultados evidencian que el modelo estadístico a través de la distribución normal multivariada (y correlacionada) es adecuado para la evaluación de la EF.

Adicionalmente, la simulación también consideró casos $500 \leq N \leq 50000$ para analizar la sensibilidad en las estadísticas de las muestras generadas con respecto al número total de muestras. Para todas las series se presentaron características estadísticas similares a la distribución histórica, y el máximo error en la estimación del caudal medio fue de $0.91 \%$ para el caso de 1500 series.

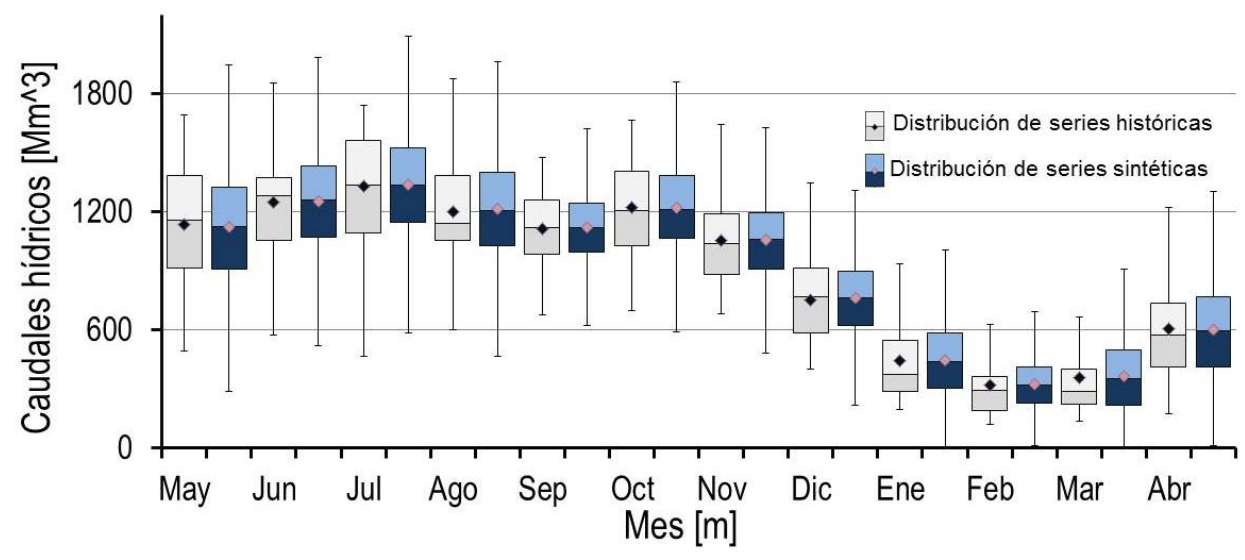

a) Distribución planta 1

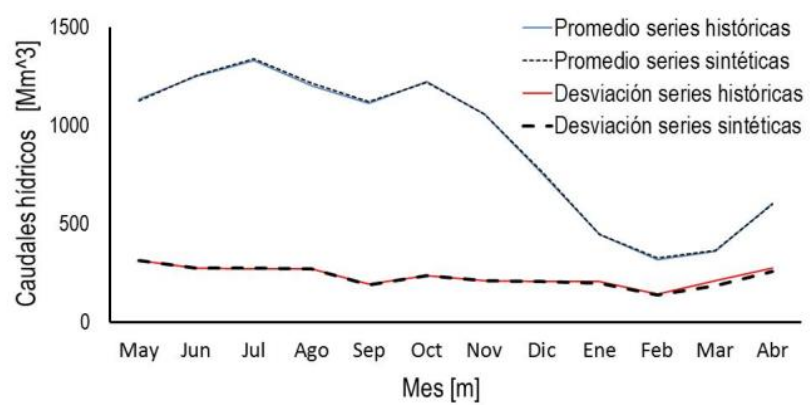

b) Promedio y desviación estándar planta 1

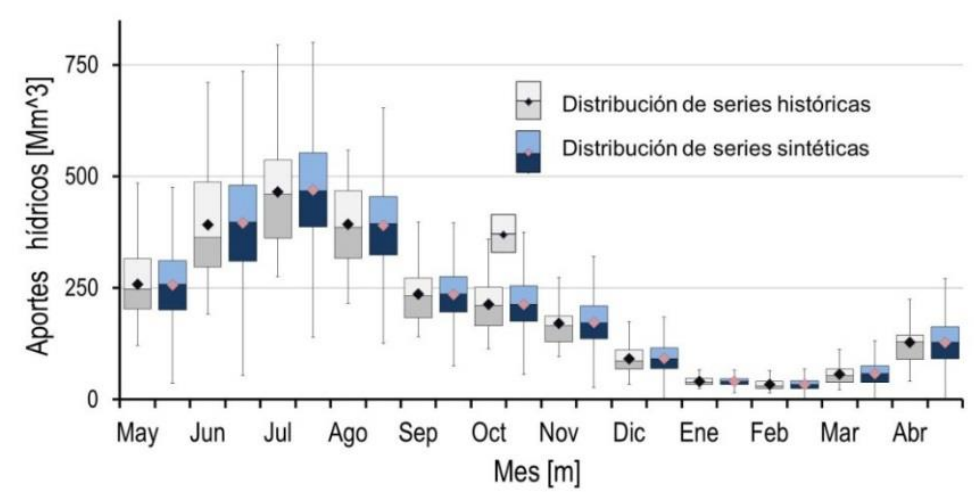

c) Distribución planta 2 


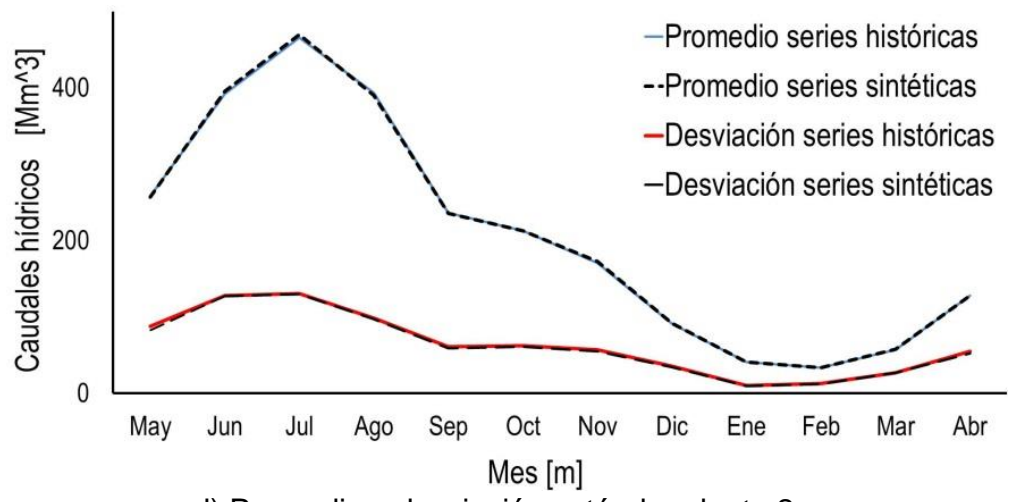

d) Promedio y desviación estándar planta 2

Fig. 5. Caudales hídricos sintéticos

\section{Comparación MCREG vs MDEF}

En esta sección se ilustra una comparación de los resultados obtenidos con la MCREG y la MDEF. Para implementar y ejecutar los modelos de optimización se utiliza el lenguaje de programación OPL, este hace parte del paquete de software CPLEX (Studio, IBM ILOG CPLEX Optimization, 2016). Para cada planta se obtienen los valores de EF de las series históricas disponibles (50 para la planta 1 y 32 para la planta 2), es decir, cada serie tiene asociada una magnitud de EF empleando el procedimiento de la Fig. 2. Para el análisis se asume que el volumen inicial $\varepsilon_{\text {inicial }}$ de cada una de las series es el $50 \%$ del volumen útil $\varepsilon_{u t i l}$. La probabilidad de excedencia de EF se presenta en la Fig. 6 a) para la planta 1 y en la Fig. 6 b) para la planta 2. La EF 95\% PE es mayor en la MDEF que en la MCREG. En los circulos rojos se resaltan las diferencias presentadas en la EF al 95\%PE.

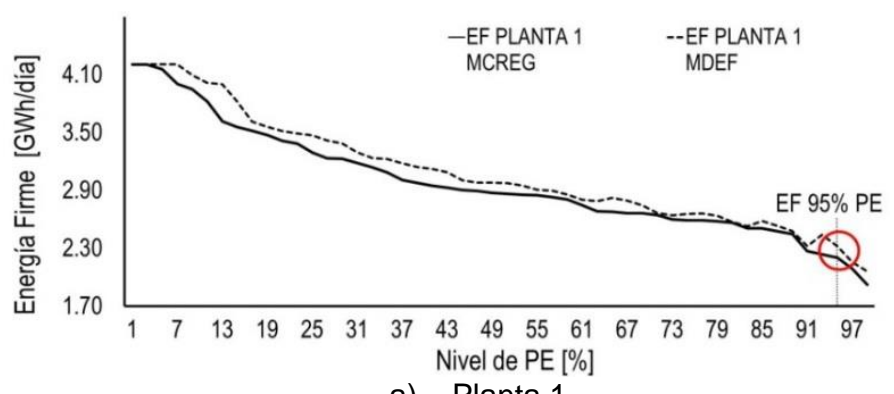

a) Planta 1

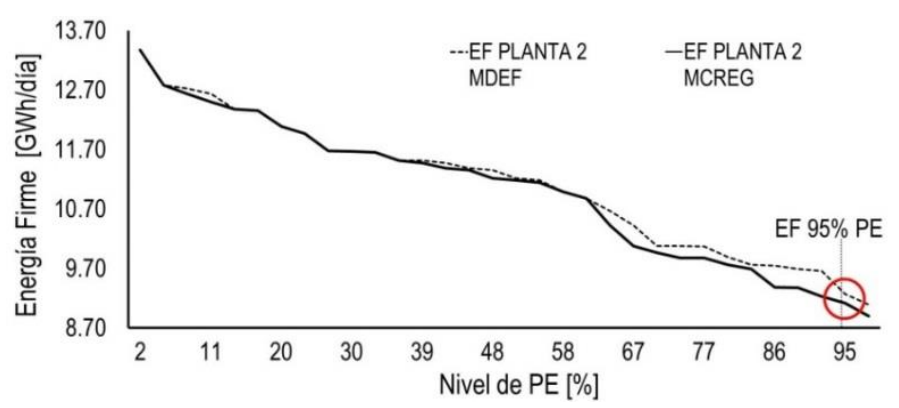

b) Planta 2

Fig. 6. Curva de distribución de probabilidad para la MCREG y la MDEF

En la Tabla 2, se muestran los resultados de la planta 1 y planta 2, al comparar la EF de la MDEF con respecto a los resultados de la MCREG. Se observa para la planta 1 un incremento de los resultados del $7.19 \%$ en la EF base y de $5.56 \%$ en la EF al $95 \%$ PE. Para la planta 2 se observa un incremento del $2.22 \%$ en la EF base y de $1.67 \%$ en la EF al $95 \%$ PE. La EF en la MDEF aumenta para ambas plantas de generación. Los incrementos se generan por la independencia en la ejecución de las series que en últimas se refleja en una relajación del modelo matemático de la MCREG en donde los volúmenes del final de un año están acoplados con los del inicio del siguiente año. En la MDEF los volúmenes están acoplados mes a mes durante el año de estudio. La MCREG implícitamente considera, para el cálculo de la EF futura, que la hidrología se repetirá en el mismo orden dado en la historia del embalse, lo cual es muy poco probable. Por el contrario, los caudales de entrada a la MDEF no consideran ningún orden cronológico de ocurrencia de las series; sin embargo, si están correlacionados mensualmente según la metodología descrita en la sección 
Proceso para generar series sintéticas. Adicionalmente, se asume una condición inicial del $50 \%$ del volumen útil en cada ejecución de cada serie hídrica, similar a lo establecido por la CREG e n su metodología original. Esto hace que el embalse alcance niveles de volumen altos en un menor tiempo y se obtenga una magnitud de EF mayor. Adicionalmente, las restricciones de curva guía mínima y curva guía máxima utilizadas en la planta 1 no alteran la tendencia de aumento de EF con la MDEF.

Tabla 2: Resultados de EF para MCREG y MDEF plantas 1 y 2

\begin{tabular}{|c|c|c|c|c|c|c|c|}
\hline Planta 1 & MCREG & $M D E F$ & $\frac{\left(E F_{M D E F}-E F_{M C R E G}\right)}{E F_{M C R E G}}$ & Planta 2 & MCREG & $M D E F$ & $\frac{\left(E F_{M D E F}-E F_{M C R E G}\right)}{E F_{M C R E G}}$ \\
\hline $\begin{array}{l}\text { EF base } \\
\text { [kWh/día] }\end{array}$ & $\begin{array}{c}1,925,14 \\
2\end{array}$ & $2,063,477$ & $7.1857 \%$ & $\begin{array}{l}\text { EF base } \\
\text { [kWh/día] }\end{array}$ & $8,895,510$ & $9,092,583$ & $2.2154 \%$ \\
\hline $\begin{array}{l}\text { EF95\%PE } \\
\text { [kWh/día] }\end{array}$ & $\begin{array}{c}2,201,82 \\
2\end{array}$ & $2,324,163$ & $5.5563 \%$ & $\begin{array}{l}\text { EF 95\%PE } \\
\text { [kWh/día] }\end{array}$ & $9,119,900$ & $9,271,800$ & $1.6656 \%$ \\
\hline
\end{tabular}

\section{Análisis de sensibilidad en el número de series sintéticas}

Típicamente, para representar adecuadamente la sensibilidad hidrológica se debe considerar un número alto de series sintéticas. En este análisis se generaron entre 500 y 50000 series de caudales con el fin de observar cambios en los resultados de la EF 95\% PE. En las Fig. 7 a) y b) se muestran las distribuciones de EF consideradas en la MDEF. El primer caso ilustrado con el menor número de series corresponde a la simulación de EF a partir de los datos históricos (50 años para la Planta 1 y 32 años para la Planta 2). Las distribuciones de la EF obtenida con la MDEF muestran que la EF se mantiene dentro de los mismos valores a pesar de incrementar el número de series. Para la planta 1 la diferencia porcentual de la mediana entre el mínimo y máximo valor con respecto al máximo valor es de $1.86 \%$ y para la planta 2 es de $5.22 \%$. La EF al 95\%PE se ilustra con círculos rojos. A partir de 500 series, la dispersión de los datos alrededor de la media es similar, donde la desviación estándar se mantiene dentro de los mismos valores. Por lo tanto, a partir de los experimentos realizados y con más de 500 series se observa que la metodología presenta menor incertidumbre, es decir, la EF al 95\%PE es constante. Esta observación es válida para ambas plantas analizadas. También resulta altamente importante mencionar que si la EF es calculada únicamente con las series históricas (50 años para la Planta 1 y 32 años para la Planta 2), es muy posible que la EF al 95\%PE esté sobreestimando su verdadero valor.



a) Planta 1

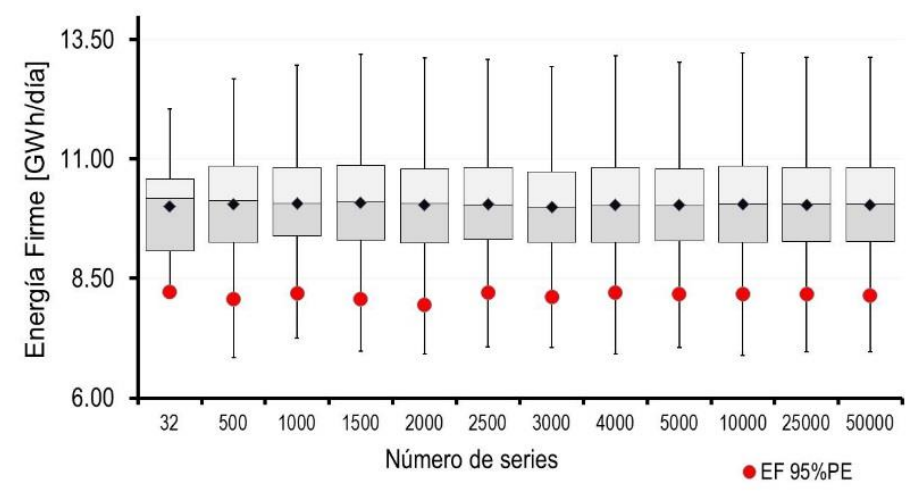

b) Planta 2

Fig. 7. Distribución de EF por el incremento de series hídricas MDEF. 
Como trabajo de investigación futuro se propone explorar el ajuste de los datos históricos de aportes hídricos con otro tipo de distribuciones o modelos estadísticos que permitan hacer una comparación para validar el comportamiento de cada una en el cálculo de EF, y finalmente determinar cuál es la representación más efectiva para las series hidrológicas.

\section{CONCLUSIONES}

Este trabajo propuso una nueva metodología para determinar la energía firme (EF) de plantas de generación hidráulicas en el contexto colombiano. Esta propuesta consistió en: i) desacoplar el modelo de la MCREG para modelar independencia de ocurrencia de las series hidricas en el cálculo de la EF, ii) construir un modelo estadístico para generar series sintéticas adecuadas que permitieron evaluar múltiples escenarios de hidrología y así poder estimar la EF con menor incertidumbre.

La distribución de los caudales hídricos históricos mostró la tendencia anual de los caudales que alimentan los embalses de cada una de las plantas de generación. La dispersión de los datos caracterizó el comportamiento de cada uno de los meses, dando como resultado final entre mayo y octubre una cresta y entre noviembre y abril un valle, esta curva coincide con las estaciones de invierno y verano en el sistema colombiano. Estos resultados permitieron realizar pronósticos de otros posibles escenarios que se pudieran presentar a través de series sintéticas empleando distribuciones normales multivariadas correlacionadas. Las series sintéticas se caracterizaron por seguir la misma tendencia de los caudales hídricos históricos. Este modelo probabilístico representa adecuadamente la incertidumbre en la hidrología de los caudales.

En este trabajo se analizaron dos plantas de generación del sistema colombiano, las cuales se eligieron por las diferencias técnicas, asociadas con la capacidad efectiva neta y con restricciones para limitar el volumen mensual $\varepsilon_{m}$. A pesar de las restricciones adicionales; el comportamiento estadístico de media, desviación estándar y dispersión de los datos es similar en ambas plantas. Lo que permite establecer que el método para el cálculo de la EF puede ser general para todas las plantas de generación hidráulica.

La MDEF muestra que se produce un incremento en la magnitud de EF con respecto a la MCREG para las dos plantas de generación, este incremento puede representar para cada planta una mayor participación en los mecanismos de capacidad de cada país que utilice como insumo la EF, esto se traduce en una mayor confiablidad del sistema de potencia cuando se presente una situación crítica de abastecimiento de agua, situación conocida en el mercado colombiano cómo periodo de escasez. Se encontró que con esta metodología es posible encontrar valores menos inciertos de EF base y EF al 95\%PE que con la metodología actual empleada por la CREG en Colombia. La razón radica en que la metodología propuesta emplea un alto número de series hidrológicas creadas con las mismas propiedades estadísticas que los registros históricos de las plantas. La propuesta de este trabajo resulta ser de fácil implementación lo que la haría atractiva para ser usada por el regulador y generadores, y así garantizar valores de EF con menores niveles de incertidumbre.

\section{AGRADECIMIENTOS}

Los autores agradecen a la Universidad de Antioquia (UdeA) por el apoyo mediante la estrategia de sostenibilidad.

\section{REFERENCIAS}

Aurelie, D., A. Edi, B. Stephanie, S. Sandrine y M. Nadia, Water Modeling in an Energy Optimization Framework - The Water-Scarce Middle East context, Applied Energy, 101(1), 268-279 (2013)

Bezerra, B., L.A. Barroso y otros cuatro autores, Measuring the Hydroelectric Regularization Capacity of the Brazilian Hydrothermal System, Power and Energy Society General Meeting IEEE, Julio 2010, Providence, USA (2010)

Choong-Kyo, H., H. Don, S. Jin-Man y P. Jong-Keun, Assessing the Impacts of Capacity Mechanisms on Generation Adequacy With Dynamic Simulations, IEEE Transactions on Power Systems, 26(4), 1788-1797 (2011)

CREG, Comisión de Regulación de Energía y Gas, Resolución CREG 071 2006, acceso: Septiembre (2017)

CREG, Comisión de Regulación de Energía y Gas, Declaración de parámetros para el cálculo de la ENFICC para la vigencia de diciembre de 2014 a noviembre de 2015, Colombia, acceso: Septiembre (2017)

CREG, Comisión de Regulación de Energía y Gas, Manual del programa HIDENFICC para calcular la energía firme ENFICC, 2007, Colombia, acceso: Septiembre (2017)

CREG, Comisión de Regulación de Energía y Gas, Resolución CREG 167 de 2017, acceso: Abril (2018)

CREG, Comisión de Regulación de Energía y Gas, Resolución CREG 201 de 2017, acceso: Abril (2018) 
Faria, E., L. A. Barroso, R. Kelman, S. Granville y M.V. Pereira, Allocation of Firm-Energy Rights Among Hydro Plants: An Aumann-Shapley Approach, IEEE Transactions on Power Systems, 24(2), 541- 551 (2009)

Hasani, M. y S.H. Hosseini, Dynamic Assessment of Capacity Investment in Electricity Market Considering Complementary Capacity Mechanisms, Energy, 36(1), 277 - 293 (2011)

Hreinsson, E. B. y L. Barroso, Defining Optimal Production Capacity in a Purely Hydroelectric Power System, IEEE 2nd International Conference on Electric Utility Deregulation, Restructuring and Power Technologies Hong Kong, China (2006)

Jaramillo, F., G. Mejía y A. Cadena, Propuesta Alternativa para la Estimación de la Energía Firme en el Mercado de Energía Eléctrica Colombiano, XIV Congreso Latino Ibero Americano de Investigación de operaciones, Cartagena de Indias, Colombia (2008)

Laurens, D. V. y H. Petra, The Impact of Electricity Market Design upon Investment under Uncertainty: The Effectiveness of Capacity Mechanisms, Utilities Policy, 16(3), 215-227 (2008)

Lennart, S., Analysis of Pricing and Volumes in Selective Capacity Markets, IEEE Transactions on Power Systems, 25(3), 1415- 1422 (2010)

MME, Ministerio de Minas y Energía y Empresa Urrá S.A. E.S.P., Situación Actual de la Central Hidroeléctrica Urrá en el Marco de la Actual Temporada Invernal, Colombia (2011)

OsInergmln, Organismo Supervisor de la Inversión en Energía y Minería, Resolución de Concejo Directivo - Oslnergmln 0362015 OS/CD, Perú (2015)

Studio, IBM ILOG CPLEX Optimization, Modeling with OPL, United State (2015)

UPME, Unidad de Planeación Minero Energética, Informe Mensual de Variables de Generación y del Mercado Eléctrico Colombiano, Noviembre de 2016 Subdirección de Energía Eléctrica (2017)

Yagi, G.D., Programação Dinâmica Aplicada ao Cálculo da Energia Firme de Usinas Hidrelétricas, Universidade Federal de Juiz de Fora, Programa de Pós-Graduação em Engenharia Elétrica (2012) 